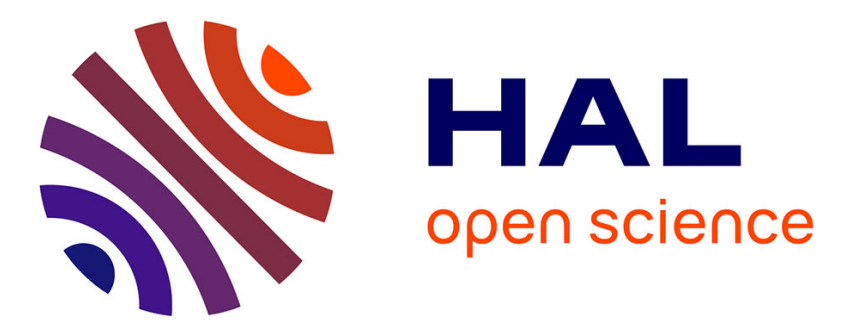

\title{
ViP-CSI Virtual Phantom Chemical Shift Imaging
}

Jie Liu, Ahmad Karfoul, Longyu Jiang, Huazhong Shu, Hervé Saint-Jalmes, Giulio Gambarota

\section{To cite this version:}

Jie Liu, Ahmad Karfoul, Longyu Jiang, Huazhong Shu, Hervé Saint-Jalmes, et al.. ViP-CSI Virtual Phantom Chemical Shift Imaging. Applied Magnetic Resonance, 2018, 49 (4), pp.369-380. 10.1007/s00723-018-0981-6 . hal-01771213

HAL Id: hal-01771213

\section{https://hal-univ-rennes1.archives-ouvertes.fr/hal-01771213}

Submitted on 4 Oct 2018

HAL is a multi-disciplinary open access archive for the deposit and dissemination of scientific research documents, whether they are published or not. The documents may come from teaching and research institutions in France or abroad, or from public or private research centers.
L'archive ouverte pluridisciplinaire HAL, est destinée au dépôt et à la diffusion de documents scientifiques de niveau recherche, publiés ou non, émanant des établissements d'enseignement et de recherche français ou étrangers, des laboratoires publics ou privés. 


\title{
ViP CSI: Virtual Phantom Chemical Shift Imaging
}

\author{
Jie Liu ${ }^{1,2,3,4}$ • Ahmad Karfoul $2^{2,3,4}$ - Longyu Jiang ${ }^{1,4}$ • \\ Huazhong Shu ${ }^{1,4}$ • Hervé Saint-Jalmes, $2,3,4$ • Giulio \\ Gambarota ${ }^{2,3,4}$
}

Received: date / Accepted: date

\begin{abstract}
Object To apply the Virtual Phantom (ViP) method for generating reference signals, to the chemical shift imaging (CSI) technique.

Materials and methods The ViP method, previously used for generating reference images in magnetic resonance imaging (MRI), was here extended to generate reference peaks in the MR spectra acquired with a 2D-CSI sequence. Theoretical analysis was carried out to design the ViP signal. ViP-2D-CSI experiments were performed on a 4.7 Tesla MR scanner. Data analysis was carried out with the jMRUI software to fit the ViP peaks in order to determine their amplitude and frequency.

Results Based on the theoretical analysis, it was found that the ViP peak could be generated by transmitting the ViP signal only in one repetition time of the 2D-CSI sequence. The amplitude and frequency of the ViP peak could be precisely adjusted and fine-tuned. The ViP peak was uniform over all CSI voxels, both in amplitude and in frequency. Furthermore, a good stability of the ViP reference amplitude and frequency was observed.

Conclusion The ViP method provides a means to generate a reference MR peak in 2D-CSI experiments. This could be of interest for signal quantification in CSI experiments.

Keywords virtual phantom (ViP) $\cdot$ chemical shift imaging (CSI) $\cdot$ physical phantom · ViP-2D-CSI

\section{Introduction}

In the last three decades, there have been considerable advances in magnetic resonance techniques for medical applications. These techniques can be broadly classified into two

凶ie Liu

liujie.list@seu.edu.cn

1 LIST, School of Computer Science and Engineering, Southeast University, Nanjing, 210096, China 2 INSERM, U1099, Rennes, F-35000, France

3 Université de Rennes 1, LTSI, Rennes, F-35000, France

4 Centre de Recherche en Information Biomédicale sino-français (CRIBs), 35000, France

5 Centre Eugène Marquis CRLCC Eugène Marquis, Aavenue Bataille Flandres-Dunkerque 35042 Rennes Cedex, FR
\end{abstract}


categories: 1) MR imaging, which provides anatomical, structural and functional information on a whole organ, with a high spatial resolution $\left(\mathrm{mm}^{3}\right)$ and 2) MR spectroscopy, which yields metabolic information on a very limited region of interest within an organ, with a low spatial resolution $\left(10 \times \mathrm{cm}^{3}\right)$. In between these two categories, one can position the MR spectroscopic imaging (MRSI), also referred to as chemical shift imaging (CSI) technique [4]. With CSI, metabolic information can be acquired on a large volume, and with a spatial resolution that is intermediate between MR imaging and MR spectroscopy techniques. CSI applications have nowadays covered the majority of organs and tissues, including brain, prostate, breast, liver, muscle and kidneys [18].

The optimization of the CSI methodology, in terms of both data acquisition and data analysis, has been and still is a very active field for research $[9,12]$. With respect to the data analysis, for the quantification of the spectroscopy signal that the CSI provides, a reference signal is necessary. Two approaches are typically employed: the use of an internal reference signal (in brain, for instance, water or creatine) or an external reference signal, which originates from a physical phantom with a well known metabolite concentration.

An alternative approach for providing a reference signal in MR spectroscopy was proposed, for applications to high resolution NMR, by Barantin et al. [2]. This approach, also referred to as the "Electronic REference To access In vivo Concentrations" (ERETIC), consists in generating a reference signal using radiofrequency (RF) electronics and transmitting it to the receiver coil of the NMR spectrometer during the data acquisition. Many studies have employed the ERETIC technique, with applications to in vitro NMR, for instance 2D-NMR, HR-MAS NMR and solid state NMR $[10,11,20]$. However, there have been only a very limited number of studies where the ERETIC technique was implemented on MR systems dedicated to in vivo applications. In other words, only few applications of ERETIC have been employed in techniques that are used in vivo $[1,5,6,8]$, such as single voxel spectroscopy, CSI and MRI. One technique that was developed to extend the ERETIC concept to in vivo application is the Virtual Phantom (ViP) for MRI [13-15, 17].

It could be advantageous to further extend the ViP technique to CSI. As a matter of fact, in CSI : 1) internal reference signals might not be valid, as they might be affected by the pathology and 2) the acquisition of the reference signal from an external phantom needs an additional measurement. It should be noted that, in CSI experiments, it is not feasible to acquire the signal from the tissue of interest simultaneously with the signal of an external phantom, since the field of view is of limited size for time saving purposes; this is in contrast to the case of MRI where in the same measurement the field of view can include both the tissue and the external phantom. Thus, it is of interest to consider the generation of a virtual phantom for signal quantification in CSI.

To apply the ViP technique to CSI, we first provide a theoretical analysis of the 2D CSI signal; then, with the designed ViP signal, we show the feasibility of adjusting and fine-tuning the amplitude and frequency of the ViP reference peak. Finally, the evaluation of the spatial uniformity and time stability of the ViP signal is performed.

Overall, the purpose of the current study was to explore the potential of ViP to generate reference signals in 2D CSI and to implement ViP-2D-CSI on an MR system dedicated to preclinical research. 


\section{Materials and methods}

\subsection{Theory}

The MR signal $R_{\mathrm{MR}}(t)$ originating from a physical phantom can be written as:

$$
R_{\mathrm{MR}}(t)=\iint_{x, y} S_{\mathrm{MR}}(x, y, t) d x d y=\iint_{x, y}\left[\int_{v} \hat{S}_{\mathrm{MR}}(x, y, v) e^{i 2 \pi v t} d v\right] d x d y
$$

where $S_{\mathrm{MR}}(x, y, t)$ is the MR signal of the element volume and $\hat{S}_{\mathrm{MR}}(x, y, v)=\int S_{\mathrm{MR}}(x, y, t) e^{-i 2 \pi v t} d t$ is the corresponding MR spectrum. In a 2D-CSI experiment, it is necessary to localize the signal from each individual voxel. The spatial encoding is then achieved by the use of phase encoding gradients, with magnetic strength $G_{x}, G_{y}$ and time duration $T$. Therefore, the equation (1) becomes:

$$
R_{\mathrm{MR}}\left(k_{x}, k_{y}, t\right)=\int_{v}\left[\iint_{x, y} \hat{S}_{\mathrm{MR}}(x, y, v) e^{i 2 \pi\left(x k_{x}+y k_{y}\right)} d x d y\right] e^{i 2 \pi v t} d v
$$

where $k_{x}$ and $k_{y}$ are defined as $\gamma G_{x} T$ and $\gamma G_{y} T$, respectively. Thus, the MR spectrum of the voxel at the spatial position $(x, y)$ can be obtained by a 2D-FT as follows:

$$
\hat{S}_{\mathrm{MR}}(x, y, v)=\iint_{k_{x}, k_{y}} \hat{R}_{\mathrm{MR}}\left(k_{x}, k_{y}, v\right) e^{-i 2 \pi\left(x k_{x}+y k_{y}\right)} d k_{x} d k_{y}
$$

where $\hat{R}_{\mathrm{MR}}\left(k_{x}, k_{y}, v\right)=\int R_{\mathrm{MR}}\left(k_{x}, k_{y}, t\right) e^{-i 2 \pi v t} d t$ denotes the FT of the received (timedomain) signal. When considering the signal of the ViP, the same mathematical formulation can be used.Thus, similarly to equation (2), the signal of the $\operatorname{ViP}, R_{\mathrm{ViP}}\left(k_{x}, k_{y}, t\right)$, can be written as:

$$
R_{\mathrm{ViP}}\left(k_{x}, k_{y}, t\right)=\int_{v}\left[\iint_{x, y} \hat{S}_{\mathrm{ViP}}(x, y, v) e^{i 2 \pi\left(x k_{x}+y k_{y}\right)} d x d y\right] e^{i 2 \pi v t} d v
$$

where $\hat{S}_{\mathrm{ViP}}(x, y, v)$ denotes the expected ViP reference peak.

Now, the question arises: what type of external (ViP) signal needs to be transmitted to the scanner bore in order to obtain a given ViP reference peak in the CSI voxels?

Let's consider the case of a reference peak with the same amplitude and frequency in all voxels. In this case, $\hat{S}_{\mathrm{ViP}}(x, y, v)$ is independent of the spatial coordinates $(x, y)$, and thus can be written as $\hat{S}_{\mathrm{ViP}}(v)$. As a consequence, the integral in equation (4) yields a 2D Dirac distribution function in the k-space, $\delta\left(k_{x}, k_{y}\right)$, as follows:

$$
\begin{aligned}
R_{\mathrm{ViP}}\left(k_{x}, k_{y}, t\right) & =\int_{v}\left[\iint_{x, y} \hat{S}_{\mathrm{ViP}}(v) e^{i 2 \pi\left(x k_{x}+y k_{y}\right)} d x d y\right] e^{i 2 \pi v t} d v \\
& =S_{\mathrm{ViP}}(t) \iint_{x, y} e^{i 2 \pi\left(x k_{x}+y k_{y}\right)} d x d y=S_{\mathrm{ViP}}(t) \delta\left(k_{x}, k_{y}\right)
\end{aligned}
$$

where $S_{\mathrm{ViP}}(t)=\int_{v} \hat{S}_{\mathrm{ViP}}(v) e^{i 2 \pi v t} d v$ is the designed signal of ViP.

As a result, the ViP peak could be generated by transmitting the ViP signal only once in any of the repetition times of the 2D-CSI sequence. In the current study, we sought to obtain a Lorentzian reference peak $\left(\hat{S}_{\mathrm{ViP}}(v)\right)$ and thus a exponential time decay signal $\left(S_{\mathrm{ViP}}(t)\right)$ was employed. 


\subsection{CSI experiments}

CSI experiments were performed on a 4.7 Tesla MR scanner (47/40 Biospec, Bruker, Wissembourg, France) using a volume coil as a transmitter/receiver. This coil, of 72-mm innerdiameter, is referred here as to the "scanner coil". The hardware configuration of the ViP apparatus was as follows: a homemade surface coil (15-mm inner-diameter, the "ViP coil") was used for transmitting the ViP signal; this coil was fixed in the scanner bore $40 \mathrm{~cm}$ away from the scanner coil. A low quality factor of the ViP coil ensured no interference with the scanner coil. Furthermore, a waveform generator (Redstone, Tecmag Inc., Houston, TX, USA) was positioned outside the scanner room to generate the ViP signal. The ViP coil was connected to the output of the waveform generator. An external trigger signal from the MR scanner was used to ensure the synchronization between the ViP signal and the ADC (analog-to-digital converter) of the MR scanner. The time domain signal representing the ViP reference peak in the CSI spectra was designed using Mathematica (Wolfram Research, Inc.); the numerical values (as magnitude and phase) of this signal served as the input of the waveform generator. Coarse values of the current intensity to be injected in the ViP coil were obtained by electromagnetic modeling, using the Principle of Reciprocity [7]. These values allowed us to generate a ViP peak with an amplitude comparable to that of a physical phantom.

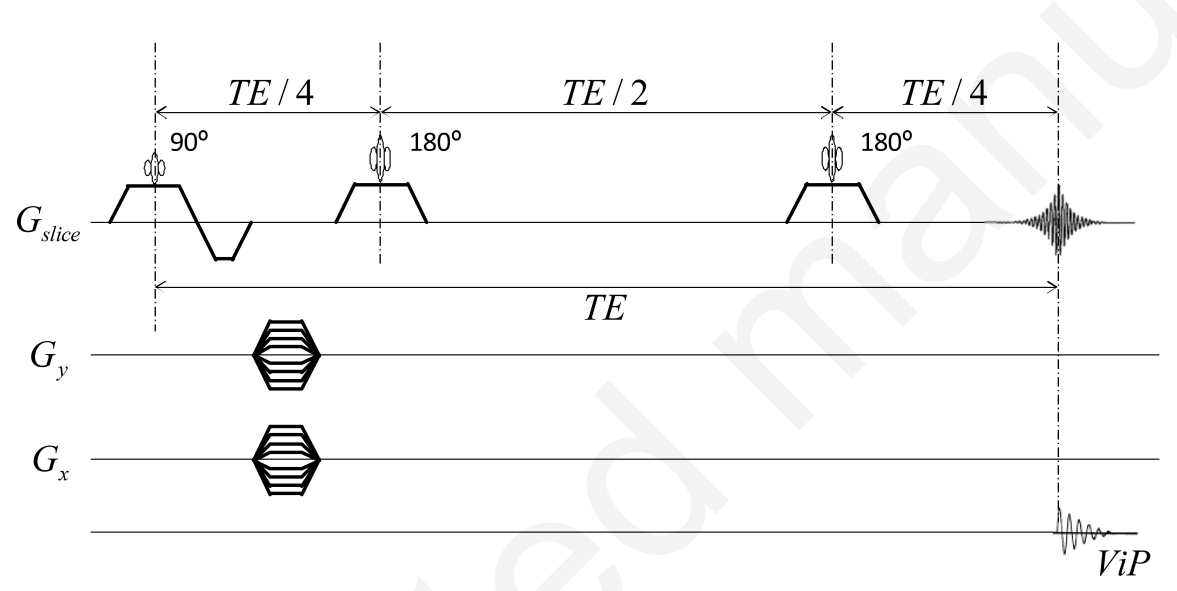

Fig. 1 The scheme of the ViP-2D-CSI sequence. A double spin-echo sequence, with interpulse delays $T E / 4$, $T E / 2$ and $T E / 4$, was employed. $T E$ is the echo time, $\mathrm{G}_{\text {slice }}$ is the slice gradient and $\mathrm{G}_{x}$ and $\mathrm{G}_{y}$ are the phase encoding gradients. The signal of the $\mathrm{ViP}$ was transmitted in synchronization with the data acquisition

CSI experiments were performed on $15 \mathrm{ml}$ cylindrical tube phantoms consisting of pure water or emulsions [16]. We refer to these phantoms as to the physical phantoms. MR images were acquired with a FLASH sequence for localization purposes and to position the CSI grid on the phantoms. The CSI measurements were performed using a 2D-spin-echo CSI sequence. The sequence diagram is shown in Fig. 1. A double spin-echo module (equivalent to the PRESS scheme [3]) was used for signal localization. CSI acquisition parameters were: repetition time $\mathrm{TR}=1200 \mathrm{~ms}$, echo time $\mathrm{TE}=30 \mathrm{~ms}$, CSI matrix size $=8 \times 8$, field of view FOV $=4 \times 4 \mathrm{~cm}^{2}$, slice thickness $=8 \mathrm{~mm}$, spatial resolution $=5 \times 5 \mathrm{~mm}^{2}$, bandwidth $=$ $2500 \mathrm{~Hz}, 1024$ data points. The ViP signal, sent by the ViP coil in synchronization with the 
MR acquisition, was acquired simultaneously with the signal from the physical phantom, as shown in Fig. 1.

Different sets of experiments were performed to investigate the performances of the ViP technique applied to 2D-CSI. These experiments are described in details in the following section.

\subsection{Data Analysis}

Data analysis was performed using the jMRUI software [19]. The AMARES algorithm was employed to fit the CSI MR spectra, in order to determine the amplitude and frequency of the resonance peaks. The peak amplitude is here defined as the area under the peak. Since the time domain signal of $\mathrm{ViP}$ was designed as a free induction decay (i.e. exponential decay), the ViP resonance peak was fitted to a Lorentzian lineshape.

After the first successful experiment for a ViP-2D-CSI, we performed additional experiments to explore ViP performances. In the first set of experiments, we investigated the feasibility of adjusting and fine-tuning the amplitude and frequency of the ViP reference peak. With respect to the amplitude of the ViP signal, five measurements were performed with different attenuation factors of the waveform generator while the frequency was kept constant. A linear regression calculation was done, and the coefficient of determination $\left(R^{2}\right)$ was obtained in order to assess the goodness of the fit. With respect to the frequency, three ViP-2D-CSI data sets were acquired with an increment of $200 \mathrm{~Hz}$, with a constant attenuation factor.

A second set of experiments was dedicated to assess the spatial un iformity and time stability of the ViP reference peak. The spatial uniformity was evaluated by fitting the ViP peak in each $\mathrm{CSI}$ voxel and by calculating the coefficient of variation $(\mathrm{CV}$, defined as the mean divided by standard deviation) of the fitted amplitudes and frequencies. The ViP time stability was determined with 5 repeated measurements. For each measurement, the normalized mean amplitude and mean frequency were obtained and their $\mathrm{CV}$ was calculated.

\section{Results}

A successful implementation of the VIP-2D-CSI is shown in Fig. 2-4. Fig. 2 illustrates the magnitude of the 64 time-domain signals of the ViP-2D-CSI experiment. The ViP signal was sent in the first TR only, and for this reason the ViP magnitude needed to be much higher than that of physical phantom, as it can be observed in Fig. 2a. A zoom-in of the first time-domain signal, consisting of both the ViP signal and the signal from the physical phantom, is shown in Fig. $2 \mathrm{~b}$ for a better visualization of both the magnitude (black line) and the real part (red line). We can observe the contribution of the physical phantom signal, as a form of oscillations, in the initial part of the decay.

The MR spectra of the same ViP-2D-CSI measurement is illustrated in Fig. 3. The water peak originating from the physical phantom can be observed at the center frequency and the $\mathrm{ViP}$ peak is at the left edge of the spectrum, as designed. The amplitude of the ViP peak appears to be uniform in all voxels.

In Fig. 4, all spectra of this ViP-2D-CSI experiment are shown. And in the spectrum example of one pixel, both the ViP peak and the water peak can be observed. 


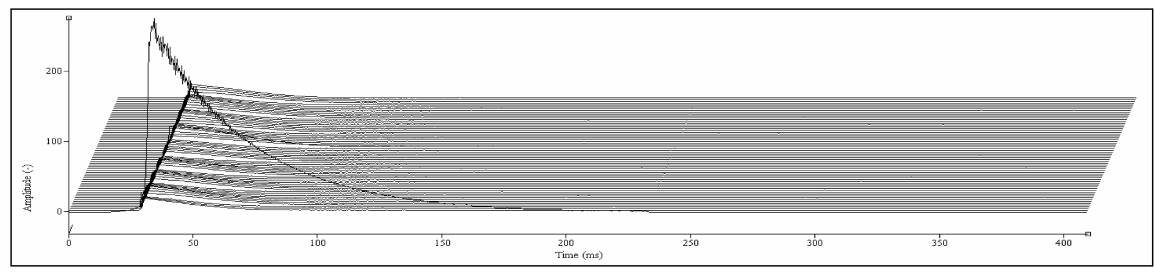

$\mathbf{a}$

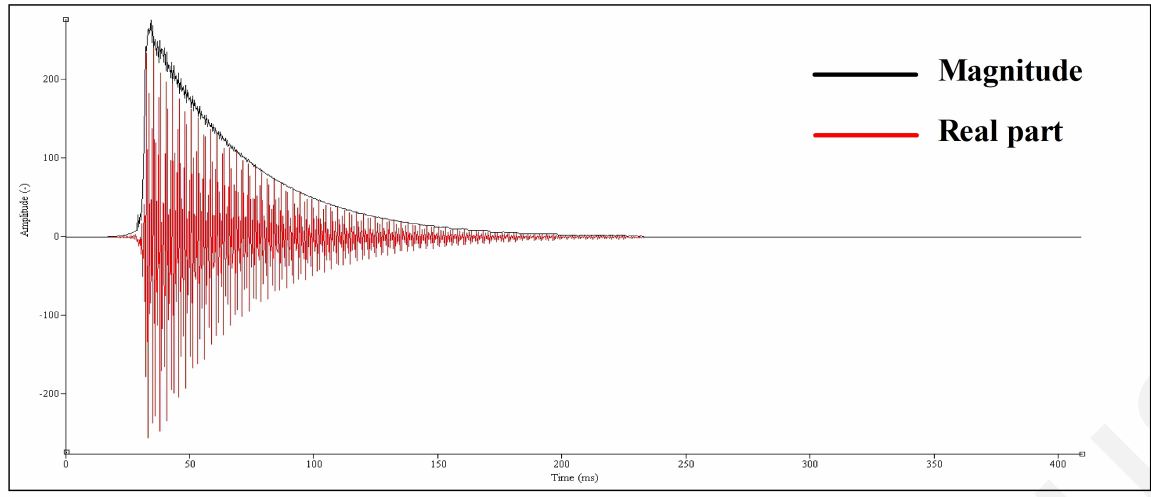

b

Fig. 2 The time-domain signals acquired in a ViP-2D-CSI measurement, for an $8 \times 8$ matrix size (repetition time $=1200 \mathrm{~ms}$, echo time $=30 \mathrm{~ms}$, acquisition points $=1024$, dwell time $=0.4 \mathrm{~ms}$ ). a. All the 64 signals are displayed in magnitude mode, for easier visualization. The ViP signal was sent only in the first of the 64 acquisitions, with a signal intensity much higher than the signal from the physical phantom. b. A detailed view of the first time-domain signal. Both the magnitude (black curve) and real part (red curve) of the signal are shown

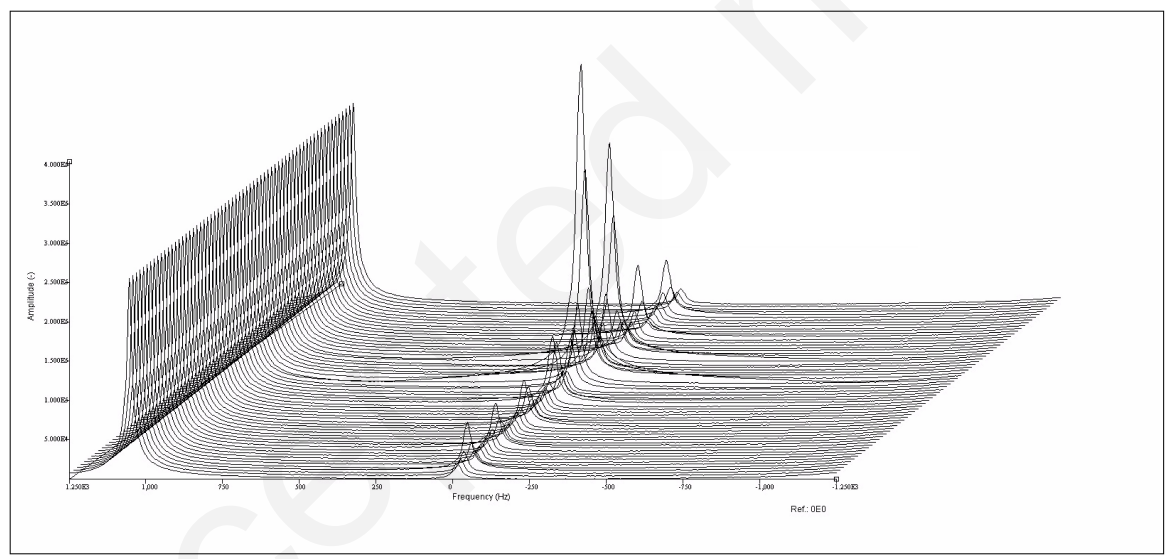

Fig. 3 The MR spectra corresponding to the ViP-2D-CSI experiment shown in Fig. 2. The ViP peak was designed to be toward the edge of the spectrum, approximately at $1 \mathrm{kHz}$ from the center frequency (the spectral bandwidth was $2.5 \mathrm{kHz}$ ). The ViP peak appears to be uniform over all voxels. The water resonance peak, originating from the physical phantom, can be observed at the center frequency 


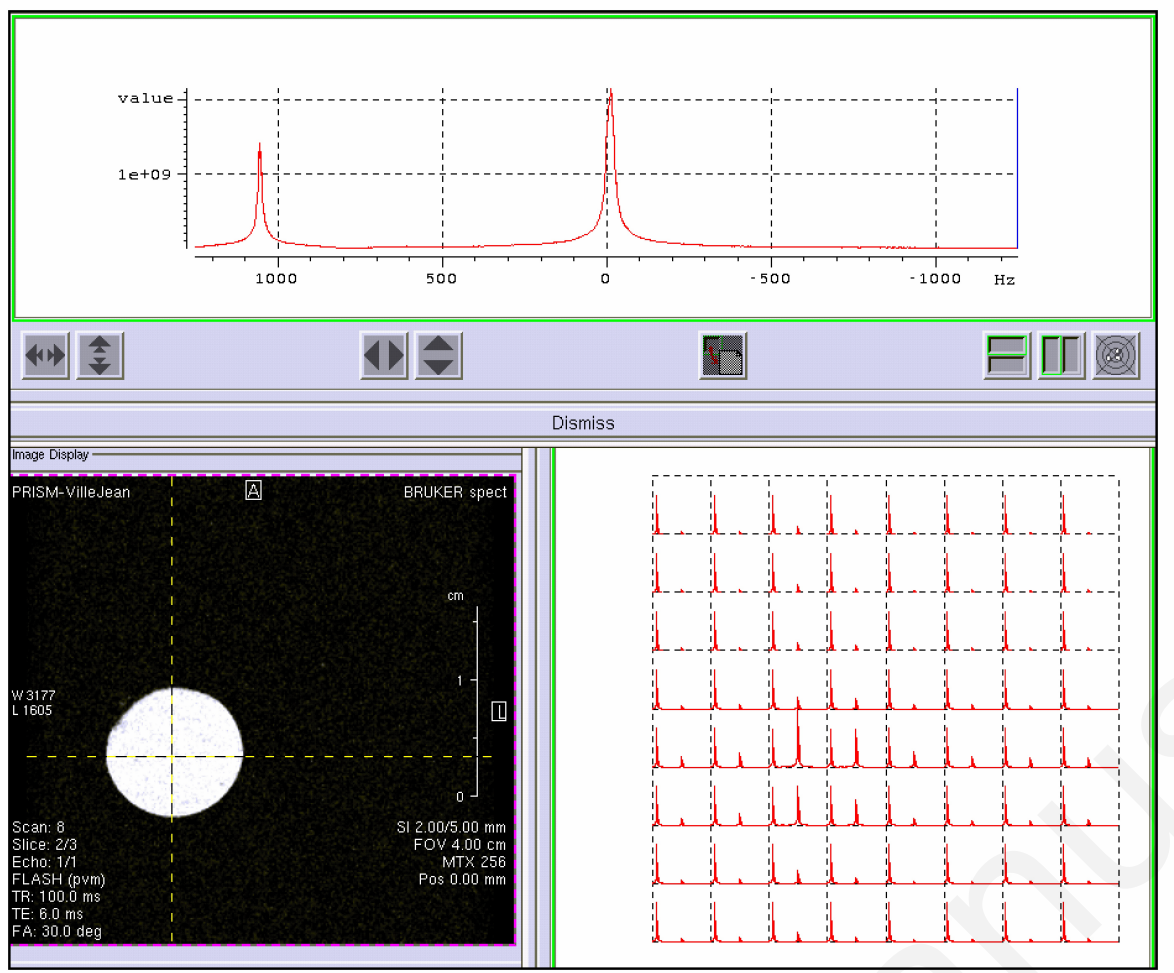

Fig. 4 A screen-shot of the CSI visualization/processing tool of the MR Bruker Biospec system. Upper panel: an example of an MR spectrum acquired in the ViP-2D-CSI experiment; the ViP peak as well as the water peak can be observed. The MR spectrum originates from the voxel whose location is illustrated by the hair-cross on the MR image (lower left panel). In the lower right panel, all spectra of the ViP-2D-CSI experiment are shown. It can be noticed that the ViP peak is present in all voxels

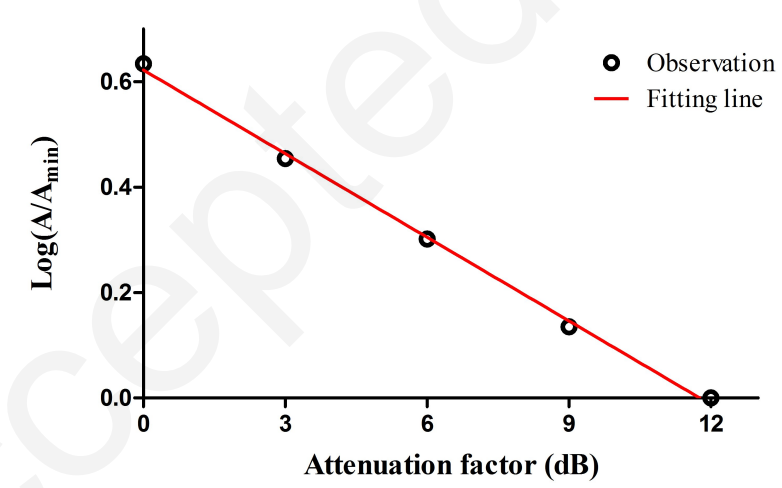

Fig. 5 The results from five ViP-2D-CSI measurements, where the ViP frequency was fixed and the attenuation factor of the waveform generator was varied from $0 \mathrm{~dB}$ to $12 \mathrm{~dB}$, with $3 \mathrm{~dB}$ increments. The amplitude of the ViP peak as a function of the attenuation factor is shown on a logarithmic scale. The continuous line indicates the linear regression curve. An excellent linearity was observed, with $R^{2}=0.9979$ 


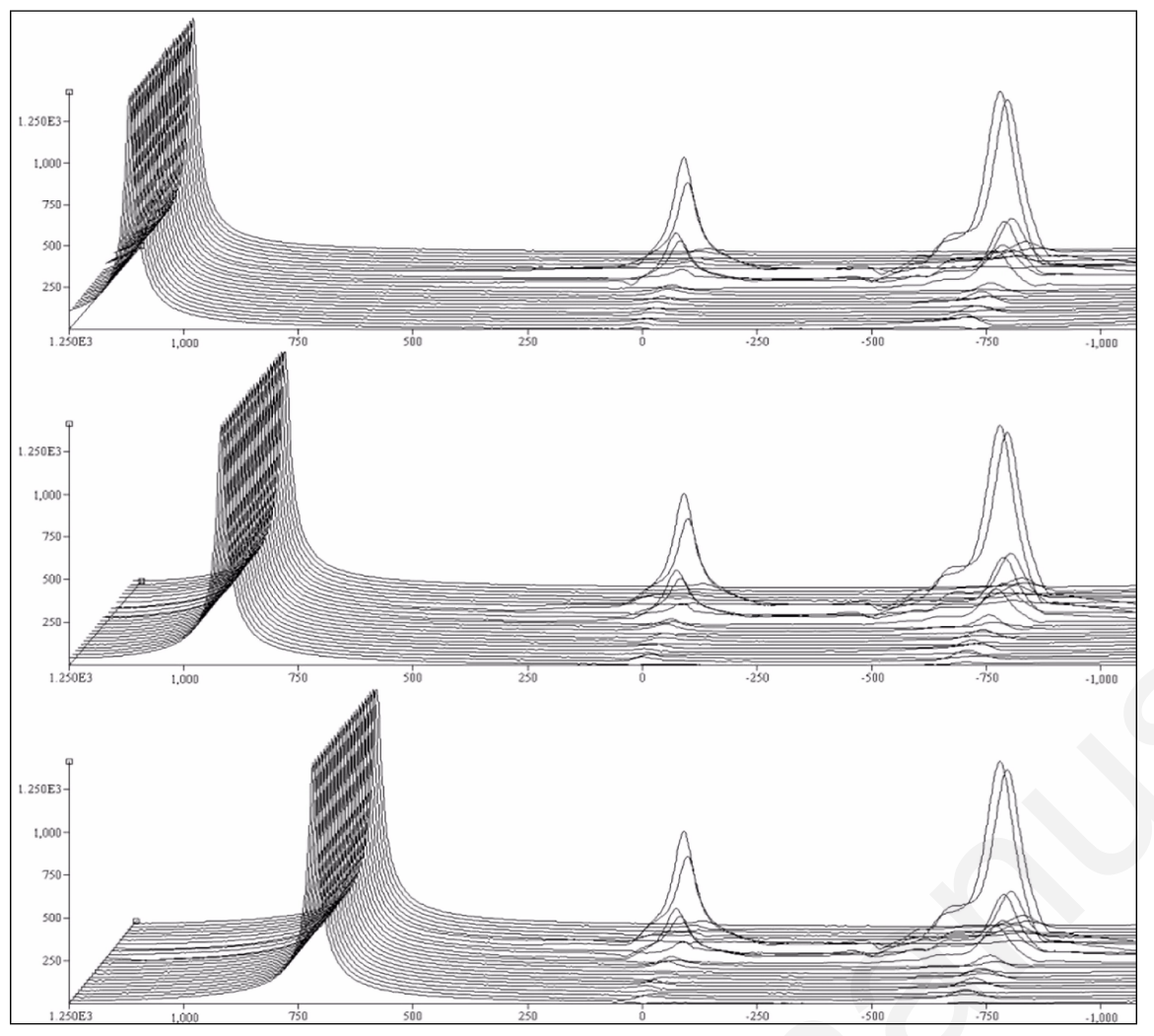

Fig. 6 The results from three ViP-2D-CSI measurements, where the attenuation factor was fixed and the ViP transmission frequency was varied with increments of $200 \mathrm{~Hz}$. For improved visualization, 24 out of the 64 voxels are shown for each ViP-2D-CSI measurement. The measured frequencies of the ViP peak in these three experiments were: $1117.7 \mathrm{~Hz}, 917.8 \mathrm{~Hz}$ and $717.8 \mathrm{~Hz}$

The feasibility of adjusting and fine-tuning the amplitude and frequency of the ViP signal are shown in Fig. 5 and Fig. 6. Fig. 5 shows the results of five ViP-2D-CSI measurements. The ViP amplitude - averaged over all voxels - was obtained for each measurement, and it is plotted in Fig. 5 as a function of the attenuation factor. An excellent linearity was observed with the coefficient of determination $R^{2}=0.9979$. Fig. 6 shows three measurements where the frequency of ViP signal was designed with an increment of $200 \mathrm{~Hz}$. The observed values of the ViP frequencies were $717.8 \mathrm{~Hz}, 917.8 \mathrm{~Hz}$ and $1117.7 \mathrm{~Hz}$, in excellent agreement with the designed increment. The frequency displacement of ViP peak had no affect on the peaks of physical phantom, as it can be observed in Fig. 6 .

Fig. 7 shows the results of the spatial uniformity and time stability measurements. The first two rows illustrate the normalized amplitude (Fig. 7a, 7c) and frequency (Fig. 7b, 7d) of the ViP signals in all voxels for two separate ViP-2D-CSI experiments. The coefficient of variation of the amplitudes and the frequencies were equal to $0.67 \%$ and $0.028 \%$ respectively, in the first experiment and $0.66 \%$ and $0.033 \%$, respectively in the second one. The third row shows the results of five measurements, repeated with the same experimental conditions. For each measurement, the time course of the mean value of the amplitude and frequency of 


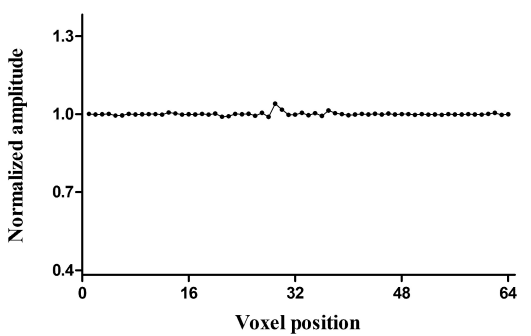

a

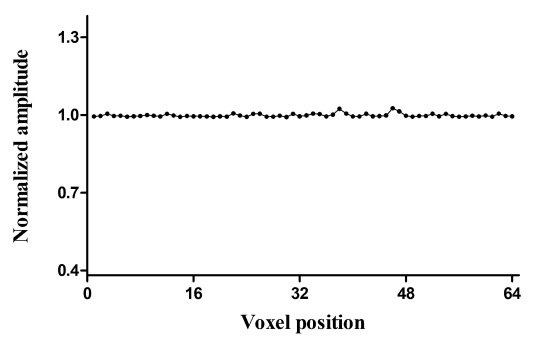

c

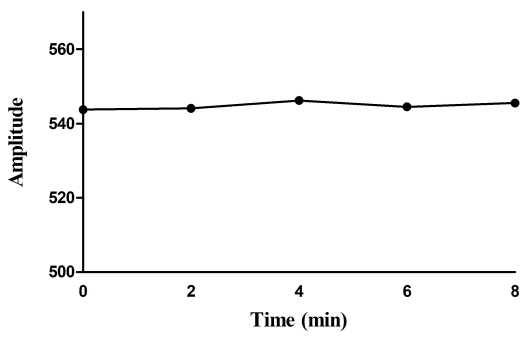

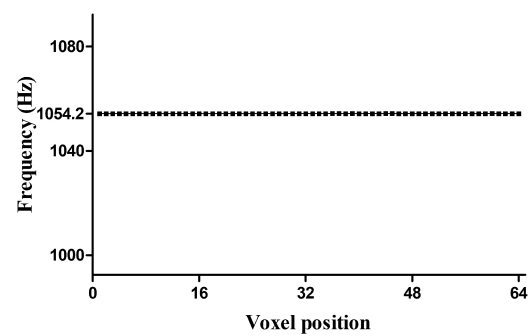

b

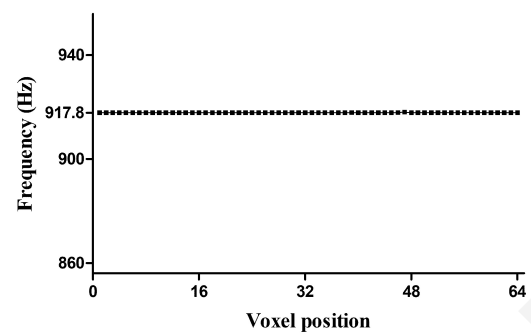

d

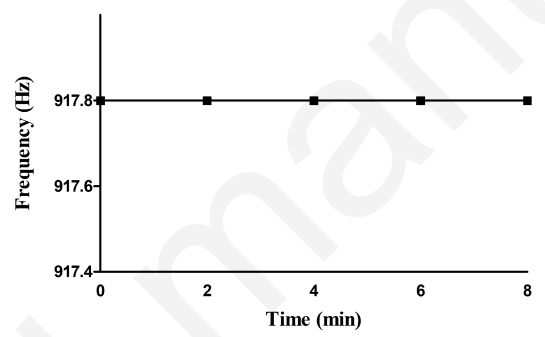

f

Fig. 7 The spatial uniformity and time stability of the ViP reference peak. Upper and center row (a-d): two separate ViP-2D-CSI experiments ( $\mathbf{a}, \mathbf{b}$ and $\mathbf{c}, \mathbf{d}$, respectively) to assess the spatial uniformity. The normalized amplitude is illustrated in $\mathbf{a}$ and $\mathbf{c}$, and the frequency in $\mathbf{b}$ and $\mathbf{d}$. Excellent uniformity was observed, with a coefficient of variation equal to $0.67 \%(\mathbf{a}), 0.028 \%$ (b), $0.66 \%$ (c) and $0.033 \%$ (d). Lower row (e, f): five ViP-2D-CSI experiments, repeated with the same experimental conditions, to assess the time stability. The mean amplitudes (the mean value of all 64 voxels for each measurement, $\mathrm{CV}=0.17 \%, \mathbf{e}$ ) and the mean frequencies (no measurable variation, $\mathbf{f}$ ) are illustrated

the ViP signal was obtained. The coefficient of variation was equal to $0.17 \%$ for the mean amplitude (Fig. 7e) and no measurable variation was found for the frequency (Fig. 7f).

\section{Discussion and Conclusion}

In the current study, we have extended the ViP approach to 2D-CSI methodology. The results of the ViP-2D-CSI experiments indicate that the virtual phantom can provide a reference signal uniform in space and stable in time. The classical 2D-CSI experiment consists of re- 
peated acquisitions of the NMR signal, for different values of the phase encoding gradients. This allows for the spatial encoding of the signal: for instance, to generate an $8 \times 8$ matrix, 64 acquisitions are needed. Based on the theoretical analysis of the 2D-CSI, we developed a strategy to generate a reference peak in all voxels, by sending the ViP signal just in one of the 64 acquisitions. This approach greatly simplifies the experimental protocol; furthermore, it is applicable to different acquisition schemes, such as elliptical and weighted CSI. As a matter of fact, in in vivo applications the full sampling of the k-space is seldom used, since it results in a long measurement scan time.

For a successful implementation of the ViP method -for both imaging and spectroscopy applications- the synchronization between the transmission of the ViP signal and the ADC acquisition window of the MR scanner is of critical importance. Without a correct synchronization, a number of artifacts will be present in the MR images or spectra. A second essential element in the ViP method is the sampling of the $\mathrm{k}$-space. It should be pointed out here a major difference between ViP-MRI and ViP-CSI. To generate a ViP image, the knowledge of the exact sampling scheme of the entire k-space is required. On the other hand, as shown in the theory section, to generate a ViP-CSI reference peak it is sufficient to send the ViP-equivalent free-induction-decay only once. Thus, for ViP-CSI the knowledge of the entire sampling scheme of $\mathrm{k}$-space is not required.

In 2D-CSI experiments, to obtain a reference signal using physical phantoms it is necessary to perform an additional measurement. This also applies to the case of the use of an internal reference, such as water for instance; as a matter of fact, typically 2D-CSI is performed with water-suppression modules, to eliminate the deleterious effects of the water resonance, which is $10^{4}$ times greater than the metabolite resonances. In addition, the internal reference signal might be affected by the pathology, thus it could yield a bias in the quantification. The proposed ViP method eliminates the need of a second measurement; furthermore, in contrast to the physical phantom, it provides a reference signal in all voxels, acquired simultaneously with the signal of interest. There are a number of useful additional features of the ViP approach: it is possible to adjust and fine-tune the amplitude and frequency of the ViP reference signal. As shown in the Result section, an excellent agreement was observed between the experimental values of amplitude and frequency and the designed ones. Furthermore, measurements of short-term time stability yielded coefficient of variation less than $0.2 \%$ in amplitude; no measurable variation was observed in frequency.

It is important to point out a number of differences between the virtual phantom and the physical phantom. First, they are not interchangeable in all situations; in other words, the virtual phantom cannot always replace the physical phantom. For instance, when developing new pulse sequences, it is of interest to test different RF pulses, gradient schemes and k-space sampling trajectories; to do so, it is necessary to acquire the MR signal originated from a physical phantom, since the ViP signal is transparent to the RF and gradient pulses.

Similarly the physical phantom cannot always replace the virtual phantom. A classical MR measurement consists of two separate processes: the spin excitation, achieved by the use of the MR-system transmission chain, and the signal reception, carried out by MR-system receiver chain. The signal of a physical phantom depends on both MR-system transmission and receiver chain; on the other hand, the signal of $\mathrm{ViP}$, its generation and transmission, is independent from the MR-system transmission chain. Thus, with the ViP signal it is possible to decouple the two processes; as a result, the ViP method can be used to selective probe the MR-system receiver chain. 


\section{References}

1. Albers, M.J., Butler, T.N., Rahwa, I., Bao, N., Keshari, K.R., Swanson, M.G., Kurhanewicz, J.: Evaluation of the ERETIC method as an improved quantitative reference for $1 \mathrm{~h} \mathrm{hr}$-mas spectroscopy of prostate tissue. Magnetic resonance in medicine 61(3), 525-532 (2009)

2. Barantin, L., Pape, A.L., Akoka, S.: A new method for absolute quantitation mrs metabolites. Magnetic resonance in medicine 38(2), 179-182 (1997)

3. Bottomley, P.A.: Spatial localization in nmr spectroscopy in vivo. Annals of the New York Academy of Sciences 508(1), 333-348 (1987)

4. Brown, T.R., Kincaid, B., Ugurbil, K.: Nmr chemical shift imaging in three dimensions. Proceedings of the National Academy of Sciences 79(11), 3523-3526 (1982)

5. Franconi, F., Chapon, C., Lemaire, L., Lehmann, V., Barantin, L., Akoka, S.: Quantitative mr renography using a calibrated internal signal (ERETIC). Magnetic resonance imaging 20(8), 587-592 (2002)

6. Heinzer-Schweizer, S., De Zanche, N., Pavan, M., Mens, G., Sturzenegger, U., Henning, A., Boesiger, P.: In-vivo assessment of tissue metabolite levels using $1 \mathrm{~h} \mathrm{mrs}$ and the electric reference to access in vivo concentrations (ERETIC) method. NMR in Biomedicine 23(4), 406-413 (2010)

7. Hoult, D.: The principle of reciprocity. Journal of Magnetic Resonance 213(2), 344-346 (2011)

8. Lee, D., Marro, K., Shankland, E., Mathis, M.: Quantitative 19f imaging using inductively coupled reference signal injection. Magnetic resonance in medicine 63(3), 570-573 (2010)

9. Lee, P., Adany, P., Choi, I.Y.: Imaging based magnetic resonance spectroscopy (mrs) localization for quantitative neurochemical analysis and cerebral metabolism studies. Analytical biochemistry 529, 4047 (2017)

10. Martínez-Bisbal, M.C., Monleon, D., Assemat, O., Piotto, M., Piquer, J., Llacer, J.L., Celda, B.: Determination of metabolite concentrations in human brain tumour biopsy samples using hr-mas and ERETIC measurements. NMR in Biomedicine 22(2), 199-206 (2009)

11. Michel, N., Akoka, S.: The application of the ERETIC method to 2d-nmr. Journal of Magnetic Resonance 168(1), 118-123 (2004)

12. Mlynárik, V., Kohler, I., Gambarota, G., Vaslin, A., Clarke, P.G., Gruetter, R.: Quantitative proton spectroscopic imaging of the neurochemical profile in rat brain with microliter resolution at ultra-short echo times. Magnetic resonance in medicine 59(1), 52-58 (2008)

13. Rondeau-Mouro, C., Kovrlija, R., Gambarota, G., Saint-Jalmes, H.: $\mu$-ViP: customized virtual phantom for quantitative magnetic resonance micro-imaging at high magnetic field. Journal of Magnetic Resonance (2016)

14. Saint-Jalmes, H., Bordelois, A., Gambarota, G.: Virtual phantom magnetic resonance imaging (ViP mri) on a clinical mri platform. Medical Physics (2017 Nov 9, DOI: 10.1002/mp.12658)

15. Saint-Jalmes, H., Eliat, P.A., Bezy-Wendling, J., Bordelois, A., Gambarota, G.: ViP mri: virtual phantom magnetic resonance imaging. Magnetic Resonance Materials in Physics, Biology and Medicine 27(5), 419-424 (2014)

16. Salvati, R., Gambarota, G.: Mri-based direct measurements of the $\mathrm{t} 2 *$ transverse relaxation time of water and lipid protons in water-lipid mixtures. Applied Magnetic Resonance 47(2), 139-148 (2016)

17. Salvati, R., Hitti, E., Bellanger, J.J., Saint-Jalmes, H., Gambarota, G.: Fat ViP mri: Virtual phantom magnetic resonance imaging of water-fat systems. Magnetic resonance imaging 34(5), 617-623 (2016)

18. Smith, I.C., Stewart, L.C.: Magnetic resonance spectroscopy in medicine: clinical impact. Progress in nuclear magnetic resonance spectroscopy 40(1), 1-34 (2002)

19. Stefan, D., Di Cesare, F., Andrasescu, A., Popa, E., Lazariev, A., Vescovo, E., Strbak, O., Williams, S., Starcuk, Z., Cabanas, M., et al.: Quantitation of magnetic resonance spectroscopy signals: the jmrui software package. Measurement Science and Technology 20(10), 104,035 (2009)

20. Ziarelli, F., Viel, S., Sanchez, S., Cross, D., Caldarelli, S.: Precision and sensitivity optimization of quantitative measurements in solid state nmr. Journal of Magnetic Resonance 188(2), 260-266 (2007) 